\title{
On Algorithmic Equivalence in Linear Fractional Programming
}

\author{
By B. Mond
}

\begin{abstract}
This paper compares the effectiveness of three published algorithms for solving the
\end{abstract} linear fractional programming problem.

Consider the problem

$$
\begin{array}{ll}
\text { Maximize } & f(\mathbf{x}) \equiv\left(\mathbf{c}^{t} \mathbf{x}+\alpha\right) /\left(\mathbf{d}^{t} \mathbf{x}+\beta\right) \\
\text { subject to } & A \mathbf{x} \leqslant \mathbf{b}, \quad \mathbf{x}>0,
\end{array}
$$

where $A \in R^{m \times n}, \mathrm{~b} \in R^{m} ; \mathrm{c}, \mathrm{d} \in R^{n} ; \alpha, \beta \in R$ and $\mathrm{d}^{\prime} \mathrm{x}+\beta>0$ for all feasible x. Charnes and Cooper [1], Isbell and Marlow [2], and Martos, in his 'simple' case, in [3] and in [4] have each given a technique for solving such a linear fractional programming problem when the constraint set is bounded. ${ }^{1}$ Wagner and Yuan [5] show that for this problem the methods of Charnes and Cooper [1] and Martos [4] are algorithmically equivalent in the sense that the two "algorithms select the same nonbasic variables to enter the next trial solution and remove the same basic variable from the current solution".

Here we compare the technique of Isbell and Marlow [2] (which involves the solution of a sequence of linear programs) to those of Charnes-Cooper and Martos. We point out that they are not equivalent, in that the Isbell-Marlow method only checks for optimality of the fractional program at points that are optimal solutions of intermediary linear programs; whereas Charnes-Cooper and Martos check for optimality of the fractional program at each step of the simplex method. Indeed, the Isbell-Marlow method, unlike the other two, may actually reach the optimal vertex and not recognize that the point is optimal. It will then proceed, or try to proceed, to another (nonoptimal) vertex. If the feasible set is bounded, the procedure will eventually return to the optimal point. If the feasible set is unbounded, the Isbell-Marlow method may fail, ${ }^{2}$ whereas the Charnes-Cooper and

Received June 14, 1979; revised November 7, 1980.

1980 Mathematics Subject Classification. Primary 90C05, 90C30.

1 Charnes-Cooper [1] and Martos [4] suggest that if the feasible set is unbounded, the problem can be regularized. As described in [4], this involves adding the constraint $x_{1}+x_{2}+\cdots+x_{n}<M$ for sufficiently large $M$.

2 We emphasize that solution procedures given by their authors for problems with bounded constraint sets are here being used (without regularization) on problems with unbounded constraint sets. Martos [4] himself gives an example in which his method fails in such a case. Our point here is that the Martos method may succeed where the Isbell-Marlow method does not, and that the Charnes-Cooper will (surprisingly) always obtain a finite optimal, if it exists, even though it is necessary, in a sense, to proceed through an infinite point before reaching the finite optimal solution. 
Martos methods will always recognize and stop at an optimal point, if such a point is reached.

We also show that for a problem with an optimal solution at a finite point and an unbounded constraint set the equivalence between the Martos and CharnesCooper methods often breaks down. In such a case, the Martos and Isbell-Marlow methods may both fail $^{3}$ whereas the Charnes-Cooper method will always yield an optimal solution.

We illustrate these differences by simple examples (compare the examples here with [4, p. 170]).

Example 1.

$$
\begin{array}{cc}
\text { Maximize } & \left(-24 x_{1}-5\right) /\left(5 x_{1}+x_{2}+1\right) \\
\text { subject to } & -x_{1}+x_{2}<1, \quad x_{1}-x_{2}<1, \quad x>0, \\
x_{1}+x_{2}<2 .
\end{array}
$$

The sequence of linear programs to be solved by the Isbell-Marlow method has the objective function

$$
R(\mathbf{x}) \equiv\left(-24-5 \lambda_{k}\right) x_{1}-\lambda_{k} x_{2}-5-\lambda_{k}, \quad \text { where } \lambda_{k}=f\left(\mathbf{x}^{k}\right)
$$

Starting with $\mathbf{x}^{1}=(0,0)$, we have $\lambda_{1}=f\left(\mathbf{x}^{1}\right)=-5$, and $R(\mathbf{x})$ becomes $x_{1}+5 x_{2}$. The simplex method proceeds to the point $(0,1)$ (the optimal solution of the fractional programming problem) and then to the point $x^{2}=(1 / 2,3 / 2)$, the optimal solution of the linear programming problem. Now, $\lambda_{2}=f\left(x^{2}\right)=-17 / 5$ and $R(\mathbf{x})$ becomes $-7 x_{1}+(17 / 5) x_{2}+8 / 5$.

Solving the linear program with this new objective function, we then return to the point $x^{3}=(0,1)$. Since this point is optimal for the linear program, we calculate $\lambda_{3}=f\left(\mathbf{x}^{3}\right)=-5 / 2$, and $R(\mathbf{x})$ becomes $-(23 / 2) x_{1}+(5 / 2) x_{2}-5 / 2$. Since at $(0,1)$, the optimal for this new linear program, $R(x)=0,(0,1)$ is optimal for the fractional program.

Note that, when starting with a new value of $\lambda_{k}$, the initial Isbell-Marlow and Martos tableaux and the methods of selecting a column to enter and leave the basis are precisely the same. At an initial basic feasible point $x^{k}$, the last line of the tableau, as recommended by Martos [3], [4], is equivalent to the tableau for the linear programming problem with objective function $\left[c-f\left(x^{k}\right) d\right]^{k} \mathbf{x}$ which is the same as the objective function [c- $\left.\lambda_{k} d\right]^{t} x$ of Isbell-Marlow. As indicated, the difference between the two methods is that Martos recalculates the last line for the new $f\left(x^{k}\right)$ at each step of the simplex method, whereas Isbell-Marlow only recalculate $\lambda_{k}$ when an optimal solution of the linear program has been obtained. Note, also, that if constraint ( 1 ) is removed from our example (so that the constraint set is now unbounded although the optimal solution is still at $(0,1))$, the Isbell-Marlow method fails ${ }^{4}$ entirely since the linear program with $R(\mathbf{x}) \equiv x_{1}+5 x_{2}$ has no solution. Here too both the Martos and Charnes-Cooper methods recognize $(0,1)$ as the optimal solution as soon as that point is reached.

\footnotetext{
${ }^{3}$ See footnote 2 .

4 See footnote 2 .
} 
Example 2.

$$
\begin{aligned}
& \max \left(-24 x_{1}-7\right) /\left(5 x_{1}+x_{2}+1\right) \\
& \text { subject to }-x_{1}+x_{2}<1, x_{1}-x_{2}<1, \quad x>0 .
\end{aligned}
$$

Here both the Martos and Isbell-Marlow methods fail ${ }^{5}$ since their methods will proceed from the starting point $(0,0)$ to the point $(1,0)$ and then along an infinite edge.

The Charnes-Cooper method [1] requires the solution of the corresponding linear program

$$
\begin{aligned}
& \max -24 y_{1}-7 t \\
& \text { subject to }-y_{1}+y_{2}-t<0, \quad y_{1}-y_{2}-t<0, \\
& \\
& 5 y_{1}+y_{2}+t=1, \quad y>0, \quad t>0 .
\end{aligned}
$$

Finite feasible $\mathbf{x}$ of Example 2 relate to feasible $(y, t)$ of the corresponding linear program by the equations

$$
x_{1}=y_{1} / t, \quad x_{2}=y_{2} / t .
$$

For corresponding finite feasible points, the values of the fractional and linear programming problems are equal. The simplex procedure generates the following sequence of basic feasible points and corresponding values of $x_{1}$ and $x_{2}$ :

$$
\begin{aligned}
\left(y_{1}, y_{2}, t, x_{1}, x_{2}\right)= & (0,0,1,0,0),(1 / 6,0,1 / 6,1,0), \\
& (1 / 6,1 / 6,0, \infty, \infty),(1 / 6,1 / 6,0, \infty, \infty),(0,1 / 2,1 / 2,0,1) .
\end{aligned}
$$

$\mathbf{x}=(0,1)$ is thus the optimal solution yielding a maximum value of $-7 / 2$.

Note that the Charnes-Cooper approach must succeed in such cases since their method requires the solution of a linear program with the same finite optimum value (as the fractional programming problem) at the corresponding finite optimal point. Moving along an infinite edge in such a fractional programming problem corresponds in the linear program to proceeding to a vertex with $t=0$. Therefore, it would appear, that, even for problems with unbounded constraint sets, regularization (and, [4, p. 172] "all the troubles regularization entails") is not necessary when using the Charnes-Cooper method for solving a linear fractional programming problem.

Department of Pure Mathematics

La Trobe University

Bundoora 3083, Victoria, Australia

1. A. Charnes \& W. W. Cooper, "Programming with linear fractional functions," Naval Res. Logist. Quart., v. 9, 1962, pp. 181-186.

2. J. R. Isbell \& W. H. MARLow, “Attrition games,” Naval Res. Logist. Quart., v. 3, 1956, pp. 1-99.

3. B. MArTos, "Hyperbolic programming," Naval Res. Logist. Quart., v. 11, 1964, pp. 135-155 (formerly appeared in Mathematical Institute, Hungarian Academy of Science, v. 5, 1960, pp. 383-406).

4. B. MArTos, Nonlinear Programming Theory and Methods, North-Holland, Amsterdam, 1975.

5. H. M. WAGNeR \& J. S. C. YUAN, "Algorithmic equivalence in linear fractional programming," Management Sci., v. 14, 1968, pp. 301-306.

\footnotetext{
${ }^{5}$ See footnote 2.
} 